\title{
Asymptotic Behavior of Solutions of Even-Order Advanced Differential Equations
}

\author{
Omar Bazighifan $\mathbb{D}^{1,2}$ and Hijaz Ahmad $\mathbb{D}^{3}$ \\ ${ }^{1}$ Department of Mathematics, Faculty of Science, Hadhramout University, Hadhramout, Yemen \\ ${ }^{2}$ Department of Mathematics, Faculty of Education, Seiyun University, Seiyun, Yemen \\ ${ }^{3}$ Department of Basic Sciences, University of Engineering and Technology, Peshawar, Pakistan \\ Correspondence should be addressed to Omar Bazighifan; o.bazighifan@gmail.com
}

Received 30 March 2020; Accepted 15 October 2020; Published 30 October 2020

Academic Editor: Praveen Agarwal

Copyright ( $\odot 2020$ Omar Bazighifan and Hijaz Ahmad. This is an open access article distributed under the Creative Commons Attribution License, which permits unrestricted use, distribution, and reproduction in any medium, provided the original work is properly cited.

In this paper, we establish the qualitative behavior of the even-order advanced differential equation $\left(a(v)\left(y^{(\kappa-1)}(v)\right)^{\beta}\right)^{\prime}+\sum_{i=1}^{j} q_{i}(v) g\left(y\left(\eta_{i}(v)\right)\right)=0, v \geq v_{0}$. The results obtained are based on the Riccati transformation and the theory of comparison with first- and second-order equations. This new theorem complements and improves a number of results reported in the literature. Two examples are presented to demonstrate the main results.

\section{Introduction}

Advanced differential equations are of practical importance, which model a phenomenon in which the rate of change of a quantity depends on present and future values of the quantity. Myschkis was the first, who discussed such equations in 1955 [1] and after him Cooke and Bellman worked further on it in 1963 [2]. These types of equations have been used in modeling of various physical and engineering phenomena. For example, population genetics [3], the study of wavelets [4], population growth [5], the field of time symmetric electrodynamics [6], neural networks [7], optimal control problems with delay [8], economics [8], dynamical systems, mathematics of networks, optimization, electrical power systems, materials, energy $j \geq 1$, etc. [9] have been studied using advanced differential equations and many approaches discussed in [10-22] can be presented for solution of such equations.

In 1980, Shah et al. [23] discussed the uniqueness and existence of the solution to nonlinear and linear such types equations, while the oscillation properties of the solution were investigated by Ladas and Stavroulakis [24], and after that, particularly in the last decade, Further refinements and improvements in the theory of advanced differential equations have been made by different researchers and it is still an active of research in engineering and applied sciences. The present paper deals with the investigation of the qualitative behavior of even-order advanced differential equation:

$$
\begin{aligned}
& \left(a(v)\left(y^{(\kappa-1)}(v)\right)^{\beta}\right)^{\prime} \\
& \left.\quad+\sum_{i=1}^{j} q_{i}(v) g\left(y\left(\eta_{i}(v)\right)\right)\right)=0, \quad v \geq v_{0},
\end{aligned}
$$

where $j \geq 1$ and $\beta$ are a quotient of odd positive integers. Throughout this work, we suppose that

$$
\begin{aligned}
& \text { C1: } a \in C^{1}\left(\left[v_{0}, \infty\right), \mathbb{R}\right), a(v)>0, a^{\prime}(v) \geq 0 \\
& \text { C2: } \quad q_{i}, \eta_{i} \in C\left(\left[v_{0}, \infty\right), \mathbb{R}\right), \quad q_{i}(v) \geq 0, \eta_{i}(v) \geq v, \\
& \lim _{v \rightarrow \infty} \eta_{i}(v)=\infty, i=1,2, \ldots, j \\
& \text { C3: } g \in C(\mathbb{R}, \mathbb{R}) \text { such that } g(x) / x^{\beta} \geq k>0, \text { for } x \neq 0
\end{aligned}
$$
and under the condition

$$
\int_{v_{0}}^{\infty} \frac{1}{a^{1 / \beta}(s)} \mathrm{d} s=\infty
$$


By a solution of (1), we mean a function $y \in C^{\kappa-1}\left[v_{y}, \infty\right), v_{y} \geq v_{0}$, which has the property $a(v)\left(y^{(\kappa-1)}(v)\right)^{\beta} \in C^{1}\left[v_{y}, \infty\right)$, and satisfies (1) on $\left[v_{y}, \infty\right)$. We consider only those solutions $y$ of (1) which satisfy $\sup \left\{|y(v)|: v \geq v_{y}\right\}>0$. A solution of (1) is called oscillatory if it has arbitrarily large zeros on $\left[v_{y}, \infty\right)$; otherwise, it is called nonoscillatory. Equation (1) is said to be oscillatory if all of its solutions are oscillatory.

\section{The Motivation of Studying this Paper}

During this decade, several works have been accomplished in the development of the oscillation theory of higher-order advanced equations by using the Riccati transformation and the theory of comparison between first- and second-order delay equations [25-39]. Further, the oscillation theory of fourth- and second-order equations has been studied and developed by using integral averaging technique and the Riccati transformation [40-45].

The study of oscillation has been carried to fractional equations in the setting of fractional operators with singular and nonsigular kernels as well (see $[46,47]$ and the references therein).

The main aim of this paper is to complement and improve the results of [48-49]. For this purpose we discuss these results.

Moaaz et al. [26] considered the fourth-order differential equation:

$$
\left(a(v)\left(y^{\prime \prime \prime}(v)\right)^{\beta}\right)^{\prime}+q(v) y^{\alpha}(\eta(v))=0,
$$

where $\beta, \alpha$ are quotients of odd positive integers.

Grace et al. [27] considered the equation

$$
\left(a(v)\left(y^{\prime \prime}(v)\right)^{\beta}\right)^{\prime \prime}+q(v) g(y(\eta(v)))=0,
$$

where $\eta(v) \leq v, \beta$ is a quotient of odd positive integers.

In particular, by using the comparison technique, the equation

$$
\left(\left(y^{(\kappa-1)}(v)\right)^{\beta}\right)^{\prime}+q(v) y^{\beta}(\eta(v))=0,
$$

has been studied by Agarwal and Grace [48], and they proved it oscillatory if

$$
\liminf _{v \longrightarrow \infty} \int_{v}^{\eta(v)}(\eta(s)-s)^{\kappa-2}\left(\int_{s}^{\infty} q(v) \mathrm{d} v\right)^{1 / \beta} \mathrm{d} s>\frac{(\kappa-2) !}{e} .
$$

Agarwal and Grace [48] extended the Riccati transformation to obtain new oscillatory criteria for (5) as condition

$$
\limsup _{v \longrightarrow \infty} v^{\beta(\kappa-1)} \int_{v}^{\infty} q(s) \mathrm{d} s>((\kappa-1) !)^{\beta} .
$$

Authors in [50] studied oscillatory behavior of (5) where $\beta=1$ and if there exists a function $\tau \in C^{1}\left(\left[v_{0}, \infty\right),(0, \infty)\right)$, also, they proved it oscillatory by using the Riccati transformation if

$$
\int_{v_{0}}^{\infty}\left(\tau(s) q(s)-\frac{(\kappa-2) !\left(\tau^{\prime}(s)\right)^{2}}{2^{3-2 \kappa} s^{\kappa-2} \tau(s)}\right) \mathrm{d} s=\infty .
$$

To prove this, we apply the previous results to the equation

$$
y^{(4)}(v)+\frac{q_{0}}{v^{4}} y(2 v)=0, \quad v \geq 1
$$

(1) By applying condition (6) in [48], we get

$$
q_{0}>25.5
$$

(2) By applying condition (7) in [49], we get

$$
q_{0}>18
$$

(3) By applying condition (8) in [50], we get

$$
q_{0}>1728 .
$$

From the above, we find the results in [49] improve results [50]. Moreover, the results in [48] improve results $[49,50]$.

Thus, the motivation in studying this paper is complement and improve results [48-50].

We shall employ the following lemmas.

Lemma 1 (see [44]). If $y^{(i)}(v)>0, i=0,1, \ldots, \kappa$, and $y^{(\kappa+1)}(v)<0$, then

$$
\frac{y(v)}{v^{\kappa} / \kappa !} \geq \frac{y^{\prime}(v)}{v^{\kappa-1} /(\kappa-1) !} .
$$

Lemma 2 (see [44]). Suppose that $y \in C^{\kappa}\left(\left[v_{0}, \infty\right),(0, \infty)\right)$, $y^{(\kappa)}$ is of a fixed sign on $\left[v_{0}, \infty\right), y^{(\kappa)}$ not identically zero and there exists a $v_{1} \geq v_{0}$ such that

$$
y^{(\kappa-1)}(v) y^{(\kappa)}(v) \leq 0,
$$

for all $v \geq v_{1}$. If we have $\lim _{v \longrightarrow \infty} y(v) \neq 0$, then there exists $v_{\theta} \geq v_{1}$ such that

$$
y(v) \geq \frac{\theta}{(\kappa-1) !} v^{\kappa-1}\left|y^{(\kappa-1)}(v)\right|,
$$

for every $\theta \in(0,1)$ and $v \geq v_{\theta}$.

Lemma 3 (see [34]). Let $\beta$ be a ratio of two odd numbers, $V>0$ and $U$ are constants. Then

$$
U x-V x^{(\beta+1) / \beta} \leq \frac{\beta^{\beta}}{(\beta+1)^{\beta+1}} \frac{U^{\beta+1}}{V^{\beta}}, \quad V>0 .
$$

Lemma 4 (see [29]). Suppose that $y$ is an eventually positive solution of (1). Then, there exist two possible cases: 
$\left(\mathbf{S}_{1}\right) \quad y(v)>0, y^{\prime}(v)>0, \quad y^{\prime \prime}(v)>0, y^{(\kappa-1)}(v)>0$, $y^{(\kappa)}(v)<0$

$\left(\mathbf{S}_{2}\right) \quad y(v)>0, \quad y^{(r)}(v)>0, \quad y^{(r+1)}(v)<0$ for all odd integer, $r \in\{1,3, \ldots, \kappa-3\}, y^{(\kappa-1)}(v)>0, y^{(\kappa)}(v)<0$

For $v \geq v_{1}$, where $v_{1} \geq v_{0}$ is sufficiently large.

\section{Comparison Theorems with Second/First- Order Equations}

Theorem 1. Assume that (2) holds. If the differential equations

$$
\begin{gathered}
\left(\frac{(\kappa-2) ! a^{1 / \beta}(v)}{\left(\theta v^{\kappa-2}\right)^{\beta}}\left(y^{\prime}(v)\right)^{\beta}\right)^{\prime}+k \sum_{i=1}^{j} q_{i}(v) y^{\beta}(v)=0, \\
y^{\prime \prime}(v)+y(v) \frac{1}{(\kappa-4) !} \int_{v}^{\infty}(\varsigma-v)^{\kappa-4} \\
\cdot\left(\frac{1}{a(\varsigma)} \int_{\varsigma}^{\infty} \sum_{i=1}^{j} q_{i}(s) \mathrm{d} s\right)^{1 / \beta} \mathrm{d} \varsigma=0 .
\end{gathered}
$$

are oscillatory. Then every solution of (1) is oscillatory.

Proof. Assume the contrary that $y$ is a positive solution of (1). Then, we can suppose that $y(v)$ and $y\left(\eta_{i}(v)\right)$ are positive for all $v \geq v_{1}$ sufficiently large. From Lemma 4, we have two possible cases $\left(\mathbf{S}_{1}\right)$ and $\left(\mathbf{S}_{2}\right)$.

Let case $\left(\mathbf{S}_{1}\right)$ holds. Using Lemma 2 , we find

$$
y^{\prime}(v) \geq \frac{\theta}{2} v^{\kappa-2} y^{(\kappa-1)}(v)
$$

for every $\theta \in(0,1)$ and for all large $v$.

Define

$$
\varphi(v):=\tau(v)\left(\frac{a(v)\left(y^{(\kappa-1)}(v)\right)^{\beta}}{y^{\beta}(v)}\right),
$$

we see that $\varphi(v)>0$ for $v \geq v_{1}$, where $\tau \in C^{1}\left(\left[v_{0}, \infty\right),(0, \infty)\right)$ and

$$
\begin{aligned}
\varphi^{\prime}(v)= & \tau^{\prime}(v) \frac{a(v)\left(y^{(\kappa-1)}(v)\right)^{\beta}}{y^{\beta}(v)} \\
& +\tau(v) \frac{\left(a\left(y^{(\kappa-1)}\right)^{\beta}\right)^{\prime}(v)}{y^{\beta}(v)} \\
& -\beta \tau(v) \frac{y^{\beta-1}(v) y^{\prime}(v) a(v)\left(y^{(\kappa-1)}(v)\right)^{\beta}}{y^{2 \beta}(v)} .
\end{aligned}
$$

Using (19) and (20), we obtain

$$
\begin{aligned}
\varphi^{\prime}(v) & \leq \frac{\tau_{+}^{\prime}(v)}{\tau(v)} \varphi(v)+\tau(v) \frac{\left(a(v)\left(y^{(\kappa-1)}(v)\right)^{\beta}\right)^{\prime}}{y^{\beta}(v)}-\beta \tau(v) \frac{\theta}{(\kappa-2) !} v^{\kappa-2} \frac{a(v)\left(y^{(\kappa-1)}(v)\right)^{\beta+1}}{y^{\beta+1}(v)} \\
& \leq \frac{\tau^{\prime}(v)}{\tau(v)} \varphi(v)+\tau(v) \frac{\left(a(v)\left(y^{(\kappa-1)}(v)\right)^{\beta}\right)^{\prime}}{y^{\beta}(v)}-\frac{\beta \theta v^{\kappa-2}}{(\kappa-2) !(\tau(v) a(v))^{1 / \beta}} \varphi(v)^{\beta+1 / \beta} .
\end{aligned}
$$

From (1) and (22), we obtain

$$
\begin{aligned}
\varphi^{\prime}(v) \leq & \frac{\tau^{\prime}(v)}{\tau(v)} \varphi(v)-k \tau(v) \frac{\sum_{i=1}^{j} q_{i}(v) y^{\beta}\left(\eta_{i}(v)\right)}{y^{\beta}(v)} \\
& -\frac{\beta \theta v^{\kappa-2}}{(\kappa-2) !(\tau(v) a(v))^{1 / \beta}} \varphi(v)^{\beta+1 / \beta} .
\end{aligned}
$$

Note that $y^{\prime}(v)>0$ and $\eta_{i}(v) \geq v$; thus, we find

$$
\begin{aligned}
\varphi^{\prime}(v) \leq & \frac{\tau^{\prime}(v)}{\tau(v)} \varphi(v)-k \tau(v) \sum_{i=1}^{j} q_{i}(v) \\
& -\frac{\beta \theta v^{\kappa-2}}{(\kappa-2) !(\tau(v) a(v))^{1 / \beta}} \varphi(v)^{\beta+1 / \beta} .
\end{aligned}
$$

If we $\operatorname{set} \tau(v)=k=1$ in (24), then we find

$$
\varphi^{\prime}(v)+\frac{\beta \theta v^{\kappa-2}}{(\kappa-2) ! a^{1 / \beta}(v)} \varphi(v)^{\beta+1 / \beta}+\sum_{i=1}^{j} q_{i}(v) \leq 0 .
$$

From [25], we can see that equation (17) is nonoscillatory, which is a contradiction.

Let case $\left(\mathbf{S}_{2}\right)$ holds. Define

$$
\psi(v):=\vartheta(v) \frac{y^{\prime}(v)}{y(v)},
$$

we see that $\psi(v)>0$ for $v \geq v_{1}$, where $\vartheta \in C^{1}\left(\left[v_{0}, \infty\right),(0, \infty)\right)$. By differentiating $\psi(v)$, we find

$$
\psi^{\prime}(v)=\frac{\vartheta^{\prime}(v)}{\vartheta(v)} \psi(v)+\vartheta(v) \frac{y^{\prime \prime}(v)}{y(v)}-\frac{1}{\vartheta(v)} \psi(v)^{2} .
$$

Now, integrating (1) from $v$ to $m$ and using $y^{\prime}(v)>0$, we find 


$$
a(m)\left(y^{(\kappa-1)}(m)\right)^{\beta}-a(v)\left(y^{(\kappa-1)}(v)\right)^{\beta}=-\int_{v}^{m} \sum_{i=1}^{j} q_{i}(s) g\left(y\left(\eta_{i}(s)\right)\right) \mathrm{d} s .
$$

By virtue of $y^{\prime}(v)>0$ and $\eta_{i}(v) \geq v$, we get

$$
\begin{aligned}
& a(m)\left(y^{(\kappa-1)}(m)\right)^{\beta}-a(v)\left(y^{(\kappa-1)}(v)\right)^{\beta} \\
& \leq-k y^{\beta}(v) \int_{v}^{u} \sum_{i=1}^{j} q_{i}(s) \mathrm{d} s .
\end{aligned}
$$

Letting $m \longrightarrow \infty$, we see that

$$
a(v)\left(y^{(\kappa-1)}(v)\right)^{\beta} \geq k y^{\beta}(v) \int_{v}^{\infty} \sum_{i=1}^{j} q_{i}(s) \mathrm{d} s,
$$

and so

$$
y^{(\kappa-1)}(v) \geq y(v)\left(\frac{k}{a(v)} \int_{v}^{\infty} \sum_{i=1}^{j} q_{i}(s) \mathrm{d} s\right)^{1 / \beta} .
$$

Integrating again from $v$ to $\infty$ for a total of $(\kappa-4)$ times, we get

$$
\begin{aligned}
& y^{\prime \prime}(v)+\frac{y(v)}{(\kappa-4) !} \int_{v}^{\infty}(\varsigma-v)^{\kappa-4} \\
& \cdot\left(\frac{k}{a(\varsigma)} \int_{\varsigma}^{\infty} \sum_{i=1}^{j} q_{i}(s) \mathrm{d} s\right)^{1 / \beta} \mathrm{d} \varsigma \leq 0
\end{aligned}
$$

From (27) and (32), we obtain

$$
\begin{aligned}
\psi^{\prime}(v) \leq & \frac{\vartheta^{\prime}(v)}{\vartheta(v)} \psi(v)-\frac{\vartheta(v)}{(\kappa-4) !} \int_{v}^{\infty}(\varsigma-v)^{\kappa-4} \\
& \cdot\left(\frac{k}{a(\varsigma)} \int_{\varsigma}^{\infty} \sum_{i=1}^{j} q_{i}(s) \mathrm{d} s\right)^{1 / \beta} \mathrm{d} \varsigma-\frac{1}{\vartheta(v)} \psi(v)^{2}
\end{aligned}
$$

If we now set $\vartheta(v)=k=1$ in (33), then we obtain

$$
\begin{aligned}
\psi^{\prime}(v)+ & \psi^{2}(v)+\frac{1}{(\kappa-4) !} \int_{v}^{\infty}(\varsigma-v)^{\kappa-4} \\
& \cdot\left(\frac{1}{a(\varsigma)} \int_{\varsigma}^{\infty} \sum_{i=1}^{j} q_{i}(s) \mathrm{d} s\right)^{1 / \beta} \mathrm{d} \varsigma \leq 0
\end{aligned}
$$

From [25], we see equation (18) is nonoscillatory, which is a contradiction. Theorem 1 is proved.

Remark 1. It is well known (see [42]) that if

$$
\int_{v_{0}}^{\infty} \frac{1}{a(v)} \mathrm{d} v=\infty
$$

$$
\liminf _{v \longrightarrow \infty}\left(\int_{v_{0}}^{v} \frac{1}{a(s)} \mathrm{d} s\right) \int_{v}^{\infty} q(s) \mathrm{d} s>\frac{1}{4},
$$

then equation

$$
\left[a(v)\left(y^{\prime}(v)\right)^{\beta}\right]^{\prime}+q(v) y^{\beta}(g(v))=0, \quad v \geq v_{0},
$$

where $\beta=1$ is oscillatory.

Based on the above results and Theorem 1, we can easily obtain the following Hille and Nehari type oscillation criteria for (1) with $\beta=1$.

Theorem 2. Let $\beta=k=1$. Assume that (2) holds. If

$$
\int_{v_{0}}^{\infty} \frac{\theta v^{\kappa-2}}{(\kappa-2) ! a(v)} \mathrm{d} v=\infty
$$

$$
\liminf _{v \longrightarrow \infty}\left(\int_{v_{0}}^{v} \frac{\theta s^{\kappa-2}}{(\kappa-2) ! a(s)} \mathrm{d} s\right) \int_{v}^{\infty} \sum_{i=1}^{j} q_{i}(s) \mathrm{d} s>\frac{1}{4}
$$

also, if

$$
\begin{aligned}
& \liminf _{v \longrightarrow \infty} v \int_{v_{0}}^{v} \frac{1}{(\kappa-4) !} \int_{v}^{\infty}(\varsigma-v)^{\kappa-4} \\
& \cdot\left(\frac{1}{a(\varsigma)} \int_{\varsigma}^{\infty} \sum_{i=1}^{j} q_{i}(s) \mathrm{d} s\right)^{1 / \beta} \mathrm{d} \varsigma \mathrm{d} v>\frac{1}{4},
\end{aligned}
$$

for some constant $\theta \in(0,1)$. Then all solution of (1) is oscillatory.

In the theorem, we compare the oscillatory behavior of (1) with the first-order differential equations:

Theorem 3. Assume that (2) holds. If the differential equations

$$
\begin{gathered}
x^{\prime}(v)+k \sum_{i=1}^{j} q_{i}(v)\left(\frac{\theta v^{\kappa-2}}{(\kappa-2) ! a^{1 / \beta}(v)}\right)^{\beta} x(\eta(v))=0, \\
z^{\prime}(v)+z(v) \frac{v}{(\kappa-4) !} \int_{v}^{\infty}(\varsigma-v)^{\kappa-4} \\
\cdot\left(\frac{k}{a(\varsigma)} \int_{\varsigma}^{\infty} \sum_{i=1}^{j} q_{i}(s) \mathrm{d} s\right)^{1 / \beta} \mathrm{d} \varsigma=0,
\end{gathered}
$$


are oscillatory, then every solution of (1) is oscillatory.

Proof. Assume the contrary that $y$ is a positive solution of (1). Then, we can suppose that $y(v)$ and $y\left(\eta_{i}(v)\right)$ are positive for all $v \geq v_{1}$ sufficiently large. From Lemma 4 , we have two possible cases $\left(\mathbf{S}_{1}\right)$ and $\left(\mathbf{S}_{2}\right)$.

In the case where $\left(\mathbf{S}_{1}\right)$ holds, from Lemma 2 , we see

$$
y(v) \geq \frac{\theta v^{\kappa-2}}{(\kappa-2) ! a^{1 / \beta}(v)}\left(a^{1 / \beta}(v) y^{(\kappa-1)}(v)\right),
$$

for every $\theta \in(0,1)$ and for all large $v$. Thus, if we set

$$
x(v)=a(v)\left(y^{(\kappa-1)}(v)\right)^{\beta}>0
$$

then we see that $\psi$ is a positive solution of the inequality.

$$
x^{\prime}(v)+k \sum_{i=1}^{j} q_{i}(v)\left(\frac{\theta v^{\kappa-2}}{(\kappa-2) ! a^{1 / \beta}(v)}\right)^{\beta} x(\eta(v)) \leq 0 .
$$

From [?, Theorem 1], we see that the equation (40) also has a positive solution, which is a contradiction.

In the case where $\left(\mathbf{S}_{2}\right)$ holds, from Lemma 1, we get

$$
y(v) \geq v y^{\prime}(v)
$$

From (32) and (45), we get

$$
\begin{aligned}
y^{\prime \prime}(v)+ & y^{\prime}(v) \frac{v}{(\kappa-4) !} \int_{v}^{\infty}(\varsigma-v)^{\kappa-4} \\
& \cdot\left(\frac{k}{a(\varsigma)} \int_{\varsigma}^{\infty} \sum_{i=1}^{j} q_{i}(s) \mathrm{d} s\right)^{1 / \beta} \mathrm{d} \varsigma \leq 0 .
\end{aligned}
$$

Now, we set

$$
z(v)=y^{\prime}(v)
$$

Thus, we find $\psi$ is a positive solution of the inequality

$$
z^{\prime}(v)+z(v) \frac{v}{(\kappa-4) !} \int_{v}^{\infty}(\varsigma-v)^{\kappa-4}
$$

$$
\left(\frac{k}{a(\varsigma)} \int_{\varsigma}^{\infty} \sum_{i=1}^{j} q_{i}(s) \mathrm{d} s\right)^{1 / \beta} \mathrm{d} \varsigma \leq 0
$$

It is well known (see [?, Theorem 1]) that the equation (41) also has a positive solution, which is a contradiction. The proof is complete.

Corollary 1. Let (2) holds. If

$$
\begin{aligned}
& \liminf _{v \longrightarrow \infty} \int_{\eta_{i}(v)}^{v} \sum_{i=1}^{j} q_{i}(s)\left(\frac{\theta v^{\kappa-2}}{(\kappa-2) ! a^{1 / \beta}(v)}\right)^{\beta} \mathrm{d} s>\frac{((\kappa-1) !)^{\beta}}{\mathrm{e}} \\
& \liminf _{v \longrightarrow \infty} \int_{\eta_{i}(v)}^{v} \frac{s}{(\kappa-4) !} \int_{v}^{\infty}(\varsigma-v)^{\kappa-4}\left(\frac{k}{a(\varsigma)} \int_{\varsigma}^{\infty} \sum_{i=1}^{j} q_{i}(s) \mathrm{d} s\right)^{1 / \beta} \mathrm{d} \varsigma \mathrm{d} s>\frac{1}{\mathrm{e}}
\end{aligned}
$$

then every solution of (1) is oscillatory.

Example 1. Let the equation

$$
y^{(4)}(v)+\frac{q_{0}}{v^{4}} y(3 v)=0, \quad v \geq 1,
$$

where $q_{0}>0$ is a constant. Note that $\beta=1, \kappa=4, a(v)=1, q(v)=q_{0} / v^{4}$, and $\eta(v)=3 v$. If we set $k=1$, then condition (38) becomes

$$
\begin{gathered}
\liminf _{v \longrightarrow \infty}\left(\int_{v_{0}}^{v} \frac{\theta s^{\kappa-2}}{(\kappa-2) ! a(s)} \mathrm{d} s\right) \int_{v}^{\infty} \sum_{i=1}^{j} q_{i}(s) \mathrm{d} s \\
=\liminf _{v \longrightarrow \infty}\left(\frac{v^{3}}{3}\right) \int_{v}^{\infty} \frac{q_{0}}{s^{4}} \mathrm{~d} s=\frac{q_{0}}{9}>\frac{1}{4}
\end{gathered}
$$

and condition (39) becomes

$$
\begin{gathered}
\liminf _{v \longrightarrow \infty} v \int_{v_{0}}^{v} \frac{1}{(\kappa-4) !} \int_{v}^{\infty}(\varsigma-v)^{\kappa-4} \\
\cdot\left(\frac{1}{a(\varsigma)} \int_{\varsigma}^{\infty} \sum_{i=1}^{j} q_{i}(s) \mathrm{d} s\right)^{1 / \beta} \mathrm{d} \varsigma \mathrm{d} v \\
=\liminf _{v \longrightarrow \infty} v\left(\frac{q_{0}}{6 v}\right)=\frac{q_{0}}{6}>\frac{1}{4} .
\end{gathered}
$$

Therefore, from Theorem 2, all solution equation (51) is oscillatory if $q_{0}>2.25$.

Remark 2. We compare our result with the known related criteria for oscillation of this equation as follows (Table 1).

Therefore, our result improves results [48-50].

Example 2. Consider a differential equation (9) where $q_{0}>0$ is a constant. Note that $\beta=1, \kappa=4, a(v)=1, q(v)=q_{0} / v^{4}$, and $\eta(v)=2 v$. If we set $k=1$, then condition (38) becomes 
TABLE 1: Comparision of results.

\begin{tabular}{lccc}
\hline The condition & $(6)$ & $(7)$ & $(8)$ \\
\hline The criterion & $q_{0}>13.6$ & $q_{0}>18$ & $q_{0}>576$ \\
\hline
\end{tabular}

$$
\frac{q_{0}}{9}>\frac{1}{4}
$$

Therefore, from Theorem 2, all solution equation (9) is oscillatory if $q_{0}>2.25$.

Remark 3. Our result improves results [48-50].

\section{Conclusion}

In this article, we study the oscillatory behavior of a class of nonlinear even-order differential equations and establish sufficient conditions for oscillation of an even-order differential equation by using the theory of comparison with first- and second-order delay equations and Riccati substitution technique.

For researchers interested in this field, and as part of our future research, there is a nice open problem which is finding new results in the following case:

$$
\int_{v_{0}}^{\infty} \frac{1}{a^{1 / \beta}(s)} \mathrm{d} s<\infty
$$

For all this, there is some research in progress.

\section{Data Availability}

No data were used to support this study.

\section{Conflicts of Interest}

The authors declare that there are no conflicts of interest associated with this publication.

\section{References}

[1] A. D. Myschkis, Lineare Differentialgleichungen mit Nacheilendem Argument, Ger-man) Deutscher Verlag der Wissenschaften, Berlin, Germany, 1955.

[2] R. Bellman and K. L. Cooke, Differential-Difference Equations, Academic Press, New York, NY, USA, 1963.

[3] D. G. Aronson and H. F. Weinberger, Nonlinear Diffusion in Population Genetics, Combustion and Nerve propagation// Lecture Notes in Mathematics, Vol. 446, Springer-Verlag, Berlin, Germany, 1975.

[4] D. W. Pravica, N. Randriampiry, and M. J. Spurr, "Theta function identities in the study of wavelets satisfying advanced differential equations," Applied and Computational Harmonic Analysis, vol. 29, no. 2, Article ID 134155, 2010.

[5] N. F. Britton, "Spatial structures and periodic traveling waves in an integro-differential reaction diffusion population model," SIAM Journal on Applied Mathematics, vol. 50, Article ID 16631688, 1990.

[6] L. S. Schulman, "Correlating arrows of time," Physical Review, vol. 7D, Article ID 28682874, 1973.

[7] M. A. Cohen and S. Grossberg, "Absolute stability of global pattern formation and parallel memory storage by competitive neural networks," IEEE Transactions on Systems, Man, and Cybernetics, vol. 13, Article ID 815826, 1983.

[8] L. S. Pontryagin, R. V. Gamkreledze, and E. F. Mischenko, The Mathematical Theory of Optimal Processes, Interscience Publishers John Wiley \& Sons, Inc, New York, NY, USA, 1962.

[9] J. K. Hale, Theory of Functional Differential Equations, Springer-Verlag, New York, NY, USA, 1977.

[10] C. Rou, Y. Whitney, X. Yousheng, and Z. Luoding, "Scalings of inverse energy transfer and energy decay in 3-d decaying isotropic turbulence with non-rotating or rotating frame of reference," Journal of Applied and Computational Mechanics, vol. 5, no. 4, pp. 639-646, 2019.

[11] H. M. Sedighi and K. H. Shirazi, "A new approach to analytical solution of cantilever beam vibration with nonlinear boundary condition," Journal of Computational and Nonlinear Dynamics, vol. 7, pp. 1-4, 2012.

[12] R. Gharechahi, M. Arab Ameri, and M. Bisheh-Niasar, "High order compact nite difference schemes for solving bratu-type equations," Journal of Applied and Computational Mechanics, vol. 5, no. 1, pp. 91-102, 2019.

[13] H. Ahmad, T. A. Khan, P. S. Stanimirović, Y. M. Chu, and I. Ahmad, "Modified variational iteration algorithm-II: convergence and applications to diffusion models," Complexity, vol. 2020, Article ID 8841718, 14 pages, 2020.

[14] H. Ahmad and T. A. Khan, "Variational iteration algorithm-i with an auxiliary parameter for wave-like vibration equations," Journal of Low Frequency Noise, Vibration and Active Control, vol. 38, no. 3-4, Article ID 11131124, 2019.

[15] E. Manconi, S. Sorokin, R. Garziera, and A. Soe-Knudsen, "Wave motion and stop-bands in pipes with helical characteristics using wave nite element analysis," Journal of Applied and Computational Mechanics, vol. 4, pp. 420-428, 2018.

[16] H. Ahmad, A. Akgül, T. A. Khan, and Y. M. Chu, "New perspective on the conventional solutions of the nonlinear time-fractional partial differential equations," Complexity, vol. 2020, Article ID 8829017, 10 pages, 2020.

[17] J.-H. He, "Hamiltonian approach to nonlinear oscillators," Physics Letters A, vol. 374, no. 23, pp. 2312-2314, 2010.

[18] H. Ahmad, A. R. Seadawy, and T. A. Khan, "Numerical solution of Korteweg-de Vries-Burgers equation by the modied variational Iteration algorithm-II arising in shallow water waves," Physica Scripta, vol. 95, no. 4, 2019.

[19] H. Ahmad and T. A. Khan, "Variational iteration algorithm I with an auxiliary parameter for the solution of differential equations of motion for simple and damped massspring systems," Noise and Vibration Worldwide, vol. 51, no. 12, p. 1220, 2020.

[20] Ji-Huan He and H. Latifizadeh, "A general numerical algorithm for nonlinear differential equations by the variational iteration method," International Journal of Numerical Methods for Heat and Fluid Flow, vol. 30, no. 11, 2020.

[21] S. Rashid, H. Ahmad, and Y. M. Chu, "On discrete fractional integral inequalities for a class of functions," Complexity, vol. 2020, Article ID 8845867, 13 pages, 2020.

[22] H. Ahmad, "Variational iteration method with an auxiliary parameter for solving differential equations of the fifth order," Nonlinear Science Letters, vol. 9, no. 1, pp. 27-35, 2018.

[23] S. M. Shah and J. Wiener, "Advanced differential equations with piecewise constant argument deviations," International Journal of Mathematics and Mathematical Sciences, vol. 6, no. 4, Article ID 671703, 1983.

[24] G. Ladas and I. P. Stavroulakis, "Oscillations caused by several retarded and advanced arguments," Journal of Differential Equations, vol. 44, no. 1, Article ID 134152, 1982. 
[25] R. P. Agarwal, S.-L. Shieh, and C.-C. Yeh, "Oscillation criteria for second-order retarded differential equations," Mathematical and Computer Modelling, vol. 26, no. 4, pp. 1-11, 1997.

[26] O. Moaaz, I. Dassios, O. Bazighifan, and A. Muhib, "Oscillation theorems for nonlinear differential equations of fourthorder," Mathematics, vol. 8, pp. 1-11, 2020.

[27] S. R. Grace, M. Bohner, and A. Liu, "Oscillation criteria for fourth-order functional differential equations," Mathematica Slovaca, vol. 63, pp. 1303-1320, 2013.

[28] O. Bazighifan and T. Abdeljawad, "Improved approach for studying oscillatory properties of fourth-order advanced differential equations with p-laplacian like operator," Mathematics, vol. 8, pp. 1-11, 2020.

[29] O. Bazighifan, H. Ahmad, and S. W. Yao, "New oscillation criteria for advanced differential equations of fourth order," Mathematics, vol. 8, pp. 1-12, 2020.

[30] R. Agarwal, S. Grace, and D. O'Regan, Oscillation Theory for Difference and Functional Differential Equations, Kluwer Acad. Publ., Dordrecht, Netherlands, 2000.

[31] B. Baculíková, J. Džurina, and J. R. Graef, "On the oscillation of higher-order delay differential equations," Journal of Mathematical Sciences, vol. 187, no. 4, pp. 387-400, 2012.

[32] O. Bazighifan and I. Dassios, "Some new oscillation results for fourth-order neutral differential equations with a canonical operator," Mathematical Problems in Engineering, vol. 2020, Article ID 8825413, , 2020.

[33] O. Bazighifan and I. Dassios, "Riccati technique and asymptotic behavior of fourth-order advanced differential equations," Mathematics, vol. 8, pp. 1-11, 2020.

[34] O. Bazighifan, E. M. Elabbasy, and O. Moaaz, "Oscillation of higher-order differential equations with distributed delay," Journal of Inequalities and Applications, vol. 55, pp. 1-9, 2019.

[35] O. Bazighifan, M. Ruggieri, and A. Scapellato, "An improved criterion for the oscillation of fourth-order differential equations," Mathematics, vol. 8, no. 4, p. 610, 2020.

[36] O. Bazighifan and M. Postolache, "Improved conditions for oscillation of functional nonlinear differential equations," Mathematics, vol. 8, no. 4, p. 552, 2020.

[37] O. Moaaz, I. Dassios, and O. Bazighifan, "Oscillation criteria of higher-order neutral differential equations with several deviating arguments," Mathematics, vol. 8, pp. 1-10, 2020.

[38] S. Grace, J. Dzurina, I. Jadlovska, and T. Li, "On the oscillation of fourth order delay differential equations," Advances in Difference Equations, vol. 118, pp. 1-15, 2019.

[39] I. Gyori and G. Ladas, Oscillation Theory of Delay Differential Equations with Applications, Clarendon Press, Oxford, UK, 1991.

[40] T. Li, B. Baculikova, J. Dzurina, and C. Zhang, "Oscillation of fourth order neutral differential equations with p-Laplacian like operators," Boundary Value Problems, vol. 56, pp. 41-58, 2014.

[41] O. Moaaz, E. M. Elabbasy, and O. Bazighifan, "On the asymptotic behavior of fourth-order functional differential equations," Advances in Difference Equations, vol. 261, pp. 1-13, 2017.

[42] Z. Nehari, "Oscillation criteria for second order linear differential equations," Transactions of the American Mathematical Society, vol. 85, pp. 428-445, 1957.

[43] P. Rehak, "How the constants in Hille-Nehari theorems depend on time scales," Advances in Difference Equations, vol. 2006, pp. 1-15, 2006.

[44] O. Bazighifan and H. Ramos, "On the asymptotic and oscillatory behavior of the solutions of a class of higher-order differential equations with middle term," Applied Mathematics Letters, vol. 107, Article ID 106431, 2020.

[45] O. Bazighifan, "An approach for studying asymptotic properties of solutions of neutral differential equations," Symmetry, vol. 12, no. 4, p. 555, 2020.

[46] A. Fernandez, T. Abdeljawad, and D. Baleanu, "Relations between fractional models with three-parameter Mittag-Leer kernels," Advances in Difference Equations, vol. 186, 2020.

[47] B. Abdalla and T. Abdeljawad, "On the oscillation of Caputo fractional differential equations with Mittag-Leffler nonsingular kernel," Chaos, Solitons \& Fractals, vol. 127, pp. 173-177, 2019.

[48] R. P. Agarwal and S. R. Grace, "Oscillation theorems for certain functional differential equations of higher order," Mathematical and Computer Modelling, vol. 39, no. 9-10, pp. 1185-1194, 2004.

[49] R. P. Agarwal, S. R. Grace, and D. O’Regan, “Oscillation criteria for certain nth order differential equations with deviating arguments," Journal of Mathematical Analysis and Applications, vol. 262, no. 2, pp. 601-622, 2001.

[50] S. R. Grace and B. S. Lalli, "Oscillation theorems for nth order nonlinear differential equations with deviating arguments," Proceedings of the American Mathematical Society, vol. 90, no. 1, pp. 65-70, 1984. 